\title{
Initial outcomes from an autism treatment demonstration
}

\author{
Steven Evans ${ }^{1 *}$ and Debra Jude Fuller ${ }^{2}$ \\ ${ }^{1}$ Therapeutics Research Institute, Omaha, Nebraska 68131, USA \\ ${ }^{2}$ Myrrh Foundation, Baltimore, MD 21201, USA
}

\begin{abstract}
In a prior peer-reviewed published medical result, significant moderation of autistic behaviors was achieved using a single supplement, the phytochemical sulforaphane. Concomitantly there have been anecdotal case studies indicating the practical and useful application of bio-resonance devices to mitigate a variety of behaviors reflected across the autistic spectrum. In a demonstration application, we undertook to combine both of these promising approaches for a small group of autistic individuals. Consistent with prior reports and case studies, quite remarkable improvements were tabulated in this study comparable to prior trial reports. Of those attributes ranked present at a severe level, $80 \%$ showed some reduction and 39\% had significant reductions in severity. This type of research might be characterized as "grassroots" science, primarily looking to tabulate the extent of any very significant participants' improvements. Given the recognition of the dire need for interventions in Asia where autism diagnoses have confronted cultural barriers to its identification due to the associated stigma such a diagnosis may bring, this intervention may also be an attractive option in such societies as a valuable adjunct to behaviorally-based autism care because of its capacity to be provided in a completely private and confidential manner.
\end{abstract}

\section{Background}

A prior autism spectrum disorder (ASD) trial, implemented by researchers from Johns Hopkins, the teaching hospital of Harvard Medical School and others in a placebo-controlled, randomized, double-blind clinical trial design, provided a daily oral administration of the phytochemical sulforaphane (derived from broccoli sprouts) for 18 weeks to 29 young men with ASD which substantially improved behaviors compared with 15 placebo recipients [1]. Behavior was quantified by parents/caregivers by the widely accepted measure of the Aberrant Behavior Checklist [2] in addition to other evaluation forms.

Sulforaphane was selected by these researchers because it upregulates genes that protect aerobic cells against oxidative stress, inflammation, and DNA-damage, and may favorably address other cellular processes all of which are prominent and possibly mechanistic characteristics of ASD or are potentially etiologically-related factors for ASD [3-18]. Concomitantly there was negligible toxicity associated with this product.

This prior trial obtained quite significant positive results among the 29 treated autism individuals. Specifically, those receiving sulforaphane showed substantial improvements in some ASD-related behaviors. Upon discontinuation of sulforaphane, scores rose toward pretreatment levels. The authors called for more research, involving larger populations, further documentation, etc.

\section{Purpose}

The purpose of this clinical demonstration was to determine whether analogously significantly positive results would accrue similar to those obtained in the prior trial noted above by purchasing essentially the same supplement over-the-counter via the Internet and administering it in a similar manner with simply personal or family supervision.

This demonstration represents an example of grassroots science
[19]. As has been reported in the popular press, grassroots science is research that is conducted by and amongst the public. It is typically very patient-outcomes oriented. There are several reasons why such a strategy was invoked. First, although this quite safe and easily utilized supplement could possibly yield immediately significant benefits to some worthwhile subset of the ASD population, further formal and traditional research to support such usage may well languish. The supplement used is inexpensive and available over-the-counter so it may not offer as much in the way of financial rewards to the traditional commercial pharmaceutical model of drug development. Securing more worthwhile patents on a synthetic analogue of this molecule might take many years and certainly a great deal of expense.

Second, ideally it would be desirable to significantly expand the size of the next formal trial population and extend its duration for a much longer period. There is a substantial challenge in obtaining the participation of ASD-affected families given the burden this condition has on family dynamics, and incorporating a placebo arm increases the demand even more in this setting. Once these barriers were overcome, if positive outcomes continued to accrue, then even further research might be warranted. With continued successes, to create a suitable FDA-approved product, a Phase I trial would be needed, then Phase II, and then a Phase III trial. With favorable results, it would be reasonable to anticipate that this completed process might see an intervention available to the public in approximately 10 or even more years, based on historical analogues [20]. In this particular application, one reason it might take even longer is the challenge to accrue a significant population of ASD-affected families to participate, some of whom might be reluctant to provide their child with a placebo for perhaps two or more years, or who might well be challenged to even manage the

Correspondence to: Steven Evans, Therapeutics Research Institute, Omaha, Nebraska 68131-2355, USA, E-mail: sevans@gsm-usa.com

Received: May 24, 2016; Accepted: June 07, 2016; Published: June 10, 2016 
difficulty to be involved at all, since it is notoriously difficult to impose on families with an autistic child.

These possibly quite substantial delays are some of the reasons we launched a grassroots science strategy. In this application, we wished to determine whether we would demonstrate essentially the same outcomes for a group of somewhat similarly ASD-affected individuals as did the earlier trial. It is labeled a demonstration since our hope was to demonstrate analogous valuable outcomes to ASD individuals using the same supplement. For this reason, our data collection was focused simply on just how many benefitted and to what extent. Then from this core demonstration base, if similar benefits accrued, we would later expect to subsequently reach out to more and more families, again with some of them benefitting. The term grassroots science is clearly an applicable description.

\section{Methods and design}

\section{Supplement}

The non-profit Therapeutics Research Institute (TRI) of Omaha, Nebraska provided essentially the same single daily capsule supplement as matched the description given in the original research above. Our justification for this selection was simply that it had been used in the prior trial with reported substantially positive outcomes. In this demonstration, the product was purchased retail on the web and given to each participant or a supervising family member to administer. There is nothing proprietary about it, and participants in the future can easily obtain it themselves if they wish, although it was provided by TRI to participants without cost in this demonstration. The specific product was Myrosinase Activated BroccoMax by Jarrow Formulas, purchased online from the Swanson Vitamin Company. BroccoMax $^{\oplus}$ contains a standardized amount of SGS, Sulforaphane Glucosinolate (a.k.a. Glucoraphanin), and the manufacturer states that the ingredients provided in one BroccoMax ${ }^{\oplus}$ delayed-release capsule have been demonstrated in vitro to yield approximately $8 \mathrm{mg}$ of actual sulforaphane.

Also in overt similarity to the prior trial research as reported, we used the same Aberrant Behavior Checklist to assess the participants.

We did not implement a placebo group. From the grassroots science perspective described above, our singular focus was simply to tabulate the extent of any worthwhile positive outcomes in those taking essentially the same supplement.

\section{The Bio-resonance intervention}

Since identifying and tabulating any improvements in the participants' outcomes was the ultimate goal of this demonstration, we added a second intervention that might also advance behavioral improvements. There is anecdotal evidence that bio-resonance can be a useful intervention for a wide range of dysfunctions [21]. A bioresonance intervention is essentially the exposure to an individual of a series of frequencies generated by a frequency generator device where each frequency series corresponds to a particular condition or medical problem which may be relieved by that frequency set.

The reader may envision a palm-sized radio-like device that is programmed to emit selected frequencies (e.g., $7.5 \mathrm{~Hz}$, etc.). The frequency generator in this demonstration was connected to a mat (approximately 13 inches square) into which a series of specific vibrational frequencies was sent. The mat was placed under any part of a trial participant for as long as could be achieved each day. Ideally it is placed under the clients as they sleep each night. The bio-resonance device was purchased online from healthproducts2.com listed as "The Rife Professional" device on its web site. One was provided by TRI to each client for their use.

A total of nine preset frequency series was assigned to be run for each participant. These were selected to address potentially significant etiological factors that have been associated with autism. Anecdotal evidence suggests that the selected frequency sets may provide health benefits to the exposed participant regarding these possible etiologic factors. So in addition to any benefits that the supplement might provide, the bio-resonance addition attempted to ameliorate some of the underlying causative factors, potentially enhancing positive outcomes.

Nine groups of frequency series were selected to be transmitted, related to associated or possibly etiological related conditions:

$\begin{array}{ll}\text { Group \#1: } & \text { Autism } \\ \text { Group \#2: } & \text { ADHD } \\ \text { Group \#3: } & \text { Comprehensive parasites } \\ \text { Group \#4: } & \text { Heavy metal detox } \\ \text { Group \#5: } & \text { Viruses particular Epstein-Barr } \\ \text { Group \#6: } & \text { Allergies/hypersensitivity } \\ \text { Group \#7: } & \text { Systemic inflammation } \\ \text { Group \#8: } & \text { Comprehensive fungal } \\ \text { Group \#9: } & \text { Overall-well-being }\end{array}$

A schedule was provided to alternate among these transmissions during the demonstration period on a weekly basis. The motivation to add this additional intervention arises from the observation that there is a number of speculated etiologic factors that may be involved with ASD.

Potentially this added intervention might address some of these underlying etiologic factors, furthering possible positive outcomes. Although we recognized that with a single-armed design we would not be able to separate out effects due only to the supplement from effects due only to the bio-resonance device, our goal to potentially maximize positive client outcomes dominated our design choice.

\section{Enrollment population}

A public ASD Forum was presented by the authors to elicit interest and to ultimately enroll participants. A total of six participants was identified, four young children, a young adult, and a middle-aged adult. This group was comprised of male Caucasians, aged 3 to 33. Given the small size of this initial population, the results are presented for each of the participants. Due to each family's personal logistics, individuals initiated their participation in the demonstration at slightly different times.

\section{Results}

\section{Case 1: Young child \# 1}

This child's parent identified the most ASD behavioral characteristics among the core 58 given in the evaluation form as used in the Hopkins et al. trial $[1,18]$, with fully 29 attributes judged as presenting as " $3=$ severe in degree." At the end of about 26 weeks, 22 of the $29(76 \%)$ showed some improvement, with a ranking move 
from "severe" to one of " $2=$ moderately serious" or less so. Of these 22 , 11 (50\%) showed significant improvement with one attribute ranked as no longer a problem at all and 10 ranked as only a slight problem now. Conversely at the other end of the spectrum, nine of $10(90 \%)$ attributes rated " $1=$ a problem but slight in degree" at the onset were now rated " $0=$ not at all a problem," while one attribute moved from "not a problem at all" to a slight problem.

\section{Case 2: Young child \#2}

This child's parent identified 21 attributes as " $3=$ severe in degree" at the onset. After about 18 weeks, eight of the 21 (38\%) moved down to " $2=$ moderately serious," and one of the attributes (4.8\%) significantly moved from " $3=$ severe in degree" to " $0=$ not at all a problem." At the other end of the spectrum, seven of eight attributes (87.5\%) ranked " $1=$ a problem but slight in degree" went to " $0=$ not at all a problem." One of the " $3=$ severe in degree" was totally resolved (4.8\%) to " $0=$ not at all a problem." An additional nine attributes were initially ranked " $2=$ moderately serious" with five of these moving down to a "slight problem" and four moving significantly down to "not a problem at all."

\section{Case 3: Young child \#3}

This child's parent initially identified six attributes as " $3=$ severe in degree." After about 24 weeks, all six (100\%) showed improvement to " $2=$ moderately serious" or better; specifically all six $(100 \%)$ were significantly reduced to " $1=$ a problem but slight in degree." At the other end of the spectrum, 26 attributes were ranked " $1=$ a problem but slight in degree" and after 24 weeks, 15 of the $26(58 \%)$ were ranked " $0=$ not at all a problem."

\section{Case 4: Young child \#4}

This child's parent identified 12 attributes as " $3=$ severe in degree" at baseline. After 22 weeks, 10 attributes were rated lower (83\%) with two of the 12 rated significantly lower at " $1=$ a problem but slight in degree" (17\%). At baseline, 12 attributes were rated " $2=$ moderately severe" while at the end of the reporting period, six of these showed some improvement $(50 \%)$ and one of the 12 showed significant improvement as " $0=$ not at all a problem" now ( $8 \%)$.

\section{Case 5: Young adult}

This young adult's parent ranked 8 attributes at level " $3=$ severe in degree." At the end of about 28 weeks, all eight of eight (100\%) showed some improvement at " $2=$ moderately serious" or better. Moreover six of the eight (75\%) were actually reduced to " $1=$ a problem but slight in degree" or a " $0=$ not at all a problem." At the other end of the spectrum, 17 attributes were initially ranked at " $1=$ a problem but slight in degree" and after the 28 weeks, 10 of the 17 (59\%) had totally resolved to " $0=$ not at all a problem." Conversely one of the total of 58 attributes which began as "not at all a problem" moved up to "a slight problem."

\section{Case 6: Middle-aged adult}

This individual identified 17 attributes at a level " $2=$ moderately serious" and only two others at a level " $3=$ severe in degree." After 28 weeks, both of the " $3=$ severe in degree" $(100 \%)$ were reduced, one to " $2=$ moderately serious" and one significantly resolved to " $0=$ not at all a problem." Since the number of attributes ranked at level 3 was so small, we focused on the number ranked at " $2=$ moderately serious." Of these 17 ranked at " $2=$ moderately serious," all (100\%) showed a reduction. Nine of the 17 (53\%) were significantly resolved to " $0=$ not at all a problem," while eight (47\%) were reduced to "a slight problem."

\section{Discussion}

Among all individuals, there was a total of 92 attributes that were identified as moderately severe or severely affected by their ASD, and 74 attributes (80\%) saw some positive change. Of these 92 total attributes, 36 (39\%) experienced significant (positive) changes. Interestingly this compares with a reported $34 \%$ significant improvement as reported in the study we are replicating although one would anticipate some methodological differences between the two studies as to precisely how this finding was computed for each [1].

Among the young children, 47 of a total of 68 affected attributes (69\%) showed positive changes, while 20 (29\%) showed significant changes. For the adults, all 27 attributes identified in the aggregate showed some positive changes (100\%) with 16 (59\%) showing significant changes. trial

There were no reported side effects among the participants in the

\section{Summary and conclusions}

For the goal of "some improvement" from the worst levels (" $3=$ severe in degree"), there were generally quite high levels recorded $(80 \%)$. For the goal of much more significant improvement (multilevel reductions) from these worst levels, there was a much wider range of responses, with significant levels of improvement on average of $39 \%$.

We note that the data identified continued improvements even throughout the longest interval (28 weeks) of participation in this trial. Since the prior trial lasted 18 weeks, this study suggests that the upside of the positive impact of this approach may not as yet be fully demonstrated.

This study demonstrated that this intervention with a capsule a day at a cost of about $\$ 9$ per month (along with the intermittent use of the Rife bio-resonance device) yields quite remarkably worthwhile outcomes. This demonstration also showed the ease by which ASDaffected individuals could confidentially achieve noteworthy behavioral improvements.

From a grassroots science perspective, it can be strongly argued that there is no basis for the timidity of national ASD organization leaders to await some distant future formal trials to support this intervention.

\section{Future directions of research}

This initiative has encouraged us to attempt to expand the target population to ever-larger circles. We will seek funding to bring new participants onboard while maintaining the prior ones. Based on this data, our target expectations for future demonstrations suggest at least a one-step reduction in the severity-level for about $80 \%$ of each participant's itemized list of most-severely-occurring autism attributes and a two-step reduction for about 39\% of each participant's list of most severe level attributes.

We have also identified both a great need as well as an under-served population in China in particular, and Asia as a whole, in part due to the cultural barriers to identifying ASD in any family due to the associated stigma such a diagnosis may bring [22-24]. Thus an additional area of focus will be foreign populations, particularly in Asia, where autism is a stigma, and affected individuals may often be hidden than provided any help of any kind.

Quite pertinent to such populations, this demonstration validated the strategy that a private, confidential, and worthwhile intervention 
could be offered that was supported by simple family supervision without concomitant public exposure.

Thus we will pursue further demonstrations in Asian settings to assess further this application as well as further evaluate the impact these interventions may exhibit.

\section{References}

1. Singh K, Connors SL, Macklin EA, Smith KD, Fahey JW, et al. (2014) Sulforaphane treatment of autism spectrum disorder (ASD). Proc Natl Acad Sci U S A 111: 1555015555. [Crossref]

2. Marshburn EC, Aman MG (1992) Factor validity and norms for the aberrant behavior checklist in a community sample of children with mental retardation. $J$ Autism Dev Disord 22: 357-373. [Crossref]

3. Zhang Y, Kensler TW, Cho CG, Posner GH, Talalay P (1994) Anticarcinogenic activities of sulforaphane and structurally related synthetic norbornyl isothiocyanates. Proc Natl Acad Sci USA 91: 3147-3150.

4. Zhang Y, Talalay P, Cho CG, Posner GH (1992) A major inducer of anticarcinogenic protective enzymes from broccoli: isolation and elucidation of structure. Proc Natl Acad Sci U S A 89: 2399-2403. [Crossref]

5. Talalay $\mathrm{P}$ (2000) Chemoprotection against cancer by induction of phase 2 enzymes. Biofactors 12: 5-11. [Crossref]

6. Juge N, Mithen RF, Traka M (2007) Molecular basis for chemoprevention by sulforaphane: a comprehensive review. Cell Mol Life Sci 64: 1105-1127. [Crossref]

7. Baird L, Dinkova-Kostova AT (2011) The cytoprotective role of the Keap1-Nrf2 pathway. Arch Toxicol 85: 241-272. [Crossref]

8. Egner PA, Chen JG, Wang JB, Wu Y, Sun Y, et al. (2011) Bioavailability of Sulforaphane from two broccoli sprout beverages: results of a short-term, cross-over clinical trial in Qidong, China. Cancer Prev Res (Phila) 4: 384-395. [Crossref]

9. Dinkova-Kostova AT (2012) The Role of Sulfhydryl Reactivity of Small Molecules for the Activation of the KEAP1/NRF2 Pathway and the Heat Shock Response. Scientifica (Cairo) 2012: 606104. [Crossref]

10. Kensler TW, Egner PA, Agyeman AS, Visvanathan K, Groopman JD, et al. (2013) Keap1-nrf2 signaling: a target for cancer prevention by sulforaphane. Top Curr Chem 329: 163-177. [Crossref]

11. James SJ, Cutler P, Melnyk S, Jernigan S, Janak L, et al. (2004) Metabolic biomarkers of increased oxidative stress and impaired methylation capacity in children with autism. Am J Clin Nutr 80: 1611-1617. [Crossref]

12. James SJ, Rose S, Melnyk S, Jernigan S, Blossom S, et al. (2009) Cellular and mitochondrial glutathione redox imbalance in lymphoblastoid cells derived from children with autism. FASEB J 23: 2374-2383. [Crossref]

13. Giulivi C, Zhang YF, Omanska-Klusek A, Ross-Inta C, Wong S, et al. (2010) Mitochondrial dysfunction in autism. JAMA 304: 2389-2396. [Crossref]

14. Napoli E, Wong S, Hertz-Picciotto I, Giulivi C (2014) Deficits in bioenergetics and impaired immune response in granulocytes from children with autism. Pediatrics 133: e1405-e1410. [Crossref]

15. Frackowiak J, Mazur-Kolecka B, Schanen NC, Brown WT, Wegiel J (2013) The link between intraneuronal -truncated amyloid- $\beta$ peptide and oxidatively modified lipids in idiopathic autism and dup(15q11.2-q13)/autism. Acta Neuropathol Commun 1: 61. [Crossref]

16. Vargas DL, Nascimbene C, Krishnan C, Zimmerman AW, Pardo CA (2005) Neuroglial activation and neuroinflammation in the brain of patients with autism. Ann Neurol 57: 67-81. [Crossref]

17. Frye RE, Rossignol DA (2014) Treatments for biomedical abnormalities associated with autism spectrum disorder. Front Pediatr 2: 66. [Crossref]

18. Gan N, Wu YC, Brunet M, Garrido C, Chung FL, et al. (2010) Sulforaphane activate heat shock response and enhances proteasome activity through up-regulation of Hsp27. J Biol Chem 285: 35528-35536. [Crossref]

19. Sal Restivo (2005) Grassroots Science. Science, Technology, \& Society: An Encyclopedia, Oxford University Press, 2005; Oxford: 175-181.

20. Frist B, Coburn T (2016) Streamlining medicine and saving lives. WSJ 12:A15.

21. Evans S (2016) Case studies in bio-resonance applications, Therapeutics Research Institute, 2016; Omaha, NE: 3-5.

22. Sun X, Allison C, Matthews FE, Sharp SJ, Auyeung B, et al. (2013) Prevalence of autism in mainland China, Hong Kong and Taiwan: a systematic review and metaanalysis. Mol Autism 4: 7. [Crossref]

23. Honda H, Shimizu Y, Misumi K, Niimi M, Ohashi Y (1996) Cumulative incidence and prevalence of childhood autism in children in Japan. Br J Psychiatry 169: 228-235. [Crossref]

24. Sun X, Allison C (2009) A review of the prevalence of autism spectrum disorder in Asia. Res Autism Spectr Disord 4: 156-167.

Copyright: (C2016 Evans S. This is an open-access article distributed under the terms of the Creative Commons Attribution License, which permits unrestricted use, distribution, and reproduction in any medium, provided the original author and source are credited. 